\title{
Monitoring of very long-chain fatty acids levels in X-linked adrenoleukodystrophy, treated with haematopoietic stem cell transplantation and Lorenzo's Oil
}

\author{
Teresa J. Stradomska ${ }^{1}$, Katarzyna Drabko², Elżbieta Moszczyńska³, Anna Tylki-Szymańska ${ }^{4}$ \\ ${ }^{1}$ Department of Biochemistry, Radioimmunology and Experimental Medicine, Children's Memorial Health Institute, Warsaw, \\ ${ }^{2}$ Department of Paediatric Haematology, Oncology and Transplantology, Medical University of Lublin, ${ }^{3}$ Department of Endocrinolo- \\ gy and Diabetology, Children's Memorial Health Institute, Warsaw, ${ }^{4}$ Department of Paediatrics, Nutrition and Metabolic Diseases, \\ Children's Memorial Health Institute, Warsaw, Poland
}

\begin{abstract}
$X$-linked adrenoleukodystrophy is a rare, neurodegenerative peroxisomal disorder connected with mutation in the $A B C D 1$ gene, causing impairment of the peroxisomal $\beta$-oxidation process and in consequence, accumulation of very long-chain fatty acids (VLCFA) in blood and tissues. In this study we present serum very long-chain fatty acids levels during clinical course in an X-linked adrenoleukodystrophy patient after haematopoietic stem cell transplantation (HSCT) and on Lorenzo's Oil in a 11 years' period. The patient was diagnosed at the age of 8 months by family screening. The administration of $L O$ was started at 2 years of age. HSCT from a family donor was performed twice. VLCFA serum levels were detected by the GC method. Chimaerism subsequent to HSCT was also analyzed.

Increasing very long-chain fatty acids levels correlate with a decreasing chimaerism level after haematopoietic stem cell transplantation. The sequential monitoring of very long-chain fatty acids serum levels is important and useful for assessment of engraftment, graft failure or rejection.
\end{abstract}

Key words: adrenoleukodystrophy, haematopoietic stem cell transplantation, very long-chain fatty acids, VLCFA, chimaerism, Lorenzo's Oil.

\section{Introduction}

X-linked adrenoleukodystrophy (X-ALD) is the most common peroxisomal neurodegenerative disorder affecting males. The clinical spectrum ranges from rapidly progressing cerebral demyelinization or slowly progressive myelopathy to isolated adrenal insufficiency. Biochemically it is characterized by the accumulation of very long-chain fatty acids (C24:0, C26:0) in plasma and tissues. X-linked adrenoleukodystrophy results from mutations in the $A B C D 1$ gene, which encodes an $A B C$ half transporter, adrenoleukodystrophy protein (ALDP). ALD peroxisomal membrane protein imports very long-chain fatty acids (VLCFA) or very long-chain fatty acids-CoA into peroxisomes, where they are degraded by a peroxisomal $\beta$-oxidation pathway. ALDP deficiency leads to impaired VLCFA $\beta$-oxidation and the accumulation of very 
long-chain fatty acids-CoA esters in cells and body fluids. The diagnosis of X-linked adrenoleukodystrophy is based on the identification of an increased level of VLCFA in blood [1].

Lorenzo's Oil (LO) administration is an option in management of X-linked adrenoleukodystrophy patients. Lorenzo's Oil, a mixture of glyceryl trioleate and glyceryl trierucate, decreases very long-chain fatty acids levels in serum. The Oil administration has been proposed in asymptomatic patients [5].

Allogeneic haematopoietic stem cell transplantation (HSCT), if available, remains the recommended therapeutic intervention that can arrest the progress of cerebral demyelinization in X-linked adrenoleukodystrophy patients. The principle behind haematopoietic stem cell transplantation is that the donorderived cells replace the bone marrow of the recipient. Physiological very long-chain fatty acids $\beta$-oxidation takes place in the donor's leucocytes [2,6-8].

The aim of this study is to evaluate the serum VLCFA levels measured in an X-linked adrenoleukodystrophy patient during his treatment. Firstly, the patient was treated with Lorenzo's Oil; secondly, he had haematopoietic stem cell transplantation twice.

\section{Material and methods}

A male child, currently 11 years and 7 months old, was diagnosed with $\mathrm{X}$-linked adrenoleukodystrophy at the age of 8 months during family screening (his elder brother died from childhood cerebral X-linked adrenoleukodystrophy at the age of 10 years). Lorenzo's Oil was administered from the age of 2 years old. At 5 months before the haematopoietic stem cell transplantation, the Lorenzo's Oil treatment was interrupted. At the age of 4 years and 7 months at the presymptomatic stage of the disease, the first haematopoietic stem cell transplantation from a family donor was performed. The first graft was rejected after a year. The second haematopoietic stem cell transplantation from the same donor was performed at the age of 6 years when the patient was still asymptomatic. At the age of 7 years and 4 months, Lorenzo's Oil was re-administered.

\section{Analytical investigations}

Very long-chain fatty acids serum levels were monitored during the Lorenzo's Oil administration before and after haematopoietic stem cell transplantations. Serum very long-chain fatty acids, such as methyl ester derivatives were detected according to a previously described method [9].

Evaluation of chimaerism after allo-SCT by fluorescence in situ hybridization analysis (XY-FISH) was performed.

The adrenal function was evaluated by a Synacthen test; detection of cortisol and ACTH and DHEAS was also carried out.

\section{Radiological investigation}

Magnetic resonance imaging (MRI) was performed on average once a year.

\section{Results}

At the age of diagnosis (8 months), detected VLCFA parameters were C24:0/C22:0 $=1.564, \mathrm{C} 26: 0 /$

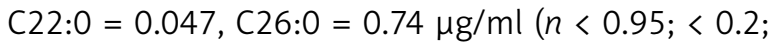
$<0.29 \mu \mathrm{g} / \mathrm{ml}$, respectively). The VLCFA of his mother were at heterozygote levels (C24:0/C22:0 = 1.076; C26:0/C22:0 = 0.024, C26:0 =0.335 $\mu \mathrm{g} / \mathrm{ml})$.

After 2 months of the first oral administration of Lorenzo's Oil with a reduction of fat in the diet, i.e. at the age of 2 years and 2 months, very long-chain fatty acids serum levels decreased to the normal level (Fig. 1A-B).

The first haematopoietic stem cell transplantation at the age of 4 years and 7 months was performed 5 months after cessation of Lorenzo's Oil therapy. Eight months after the engraftments, serum VLCFA levels decreased to heterozygote levels, mainly C24:0/C22:0. However, they subsequently increased to the levels characteristic of X-linked adrenoleukodystrophy hemizygotes, parallel with decreased chimaerism (5\% XX and 95\% XY a year after HSCT). The first graft was rejected.

The second haematopoietic stem cell transplantation was performed when the patient was 5 years and 10 months old. Immediately after the second transplantation, a rapid increase in VLCFA was observed, mainly as C26:0 concentration and C26:0/ C22:0 ratio. For 3.5 months after the transplantation, chimaerism was maintained above the $85 \%$ level and then C26:0 concentration slowly decreased to 50\%. The lowest value of C26:0 and C24:0 was obtained at chimaerism of $55 \% \mathrm{XX}$ and $45 \% \mathrm{XY}$ and CD3 detected in lymphocytes $\mathrm{T}$ was $88 \%$ and $12 \%$, respectively, 5 months after the engraftment. Since the 8.5 months after the transplantation, a repeated increase in C26:0 concentration was observed, approximately 
A

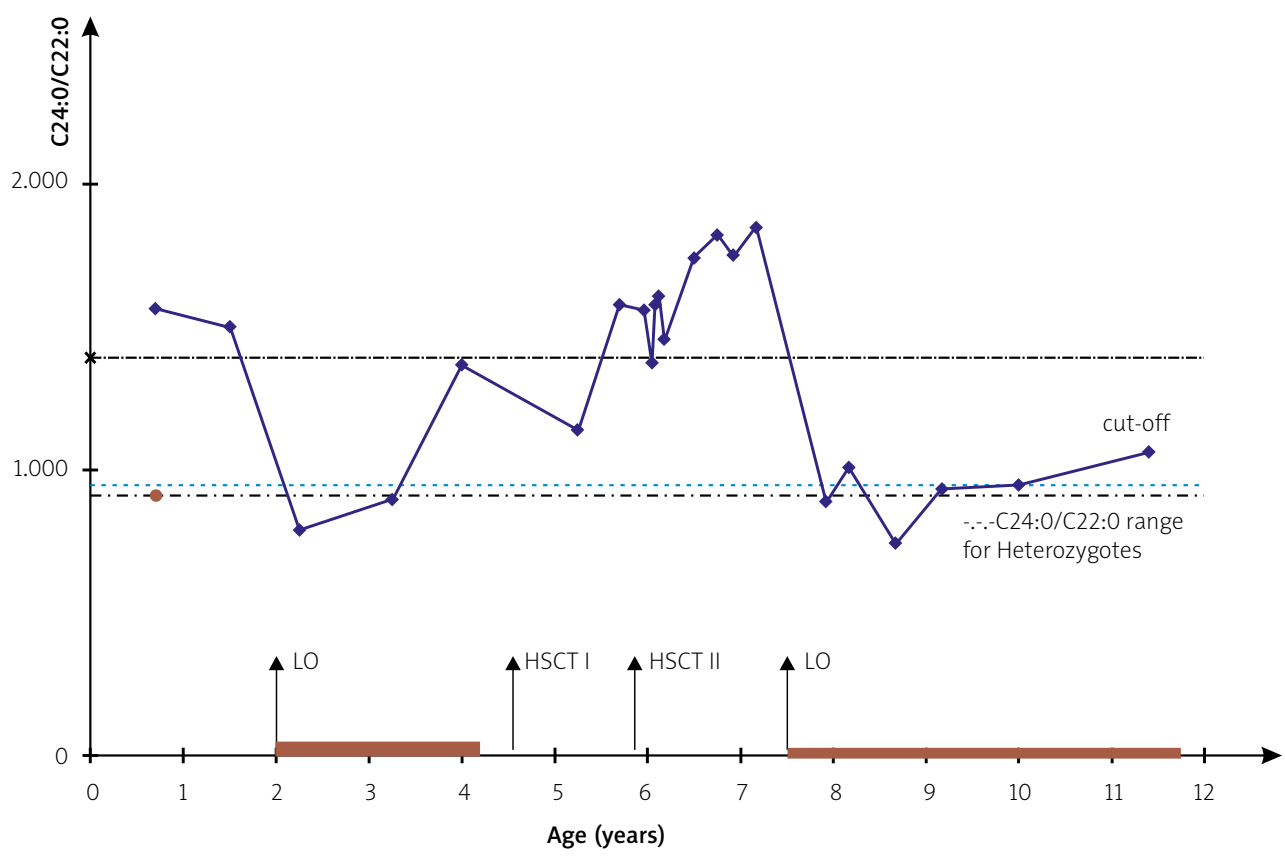

B

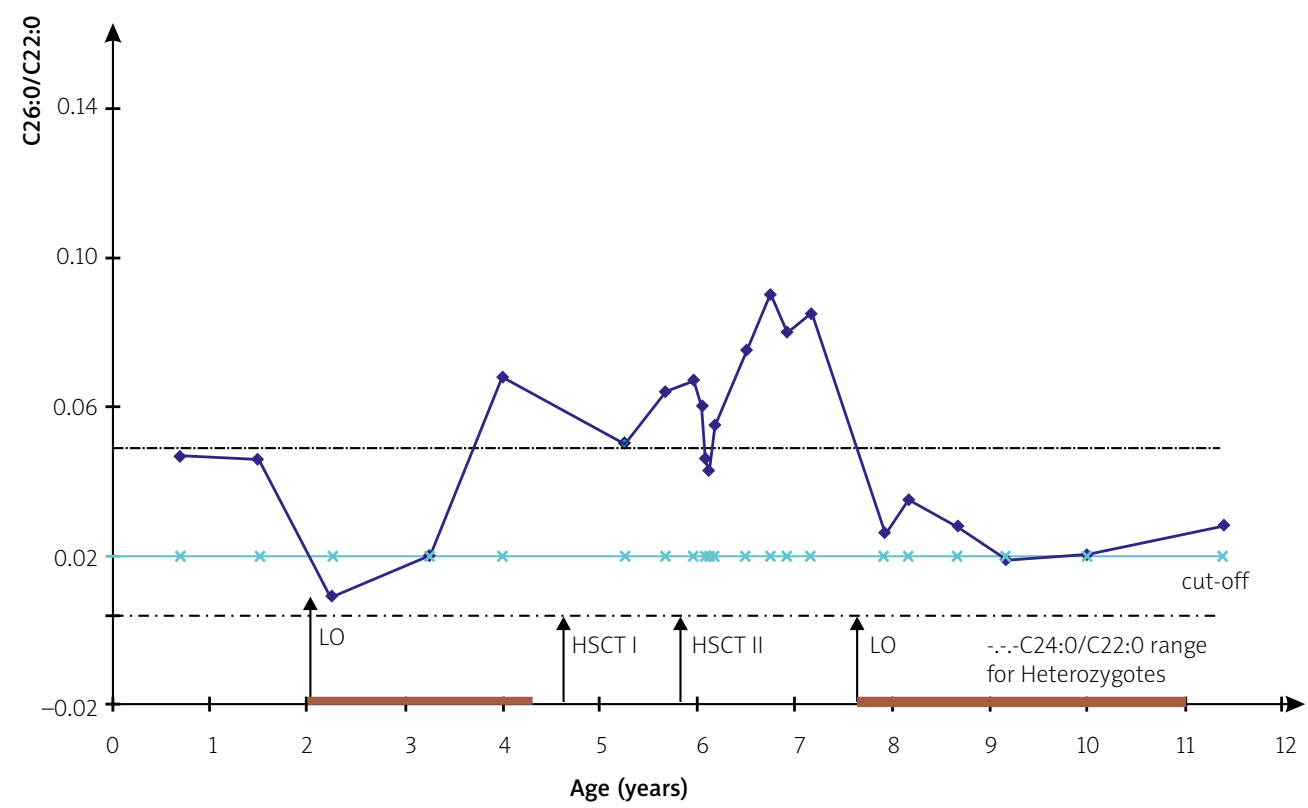

Fig. 1. The C24:0/C22:0 ratio and C26:0/C22:0 ratio in various approaches to the treatment of $X$-linked adrenoleukodystrophy in the presented patient (bold line - time of Lorenzo's Oil treatment).

$80 \%$, at chimaerism of $32 \% X X$ although detected CD3 levels were $63 \%$ XX and $37 \%$ XY. Twenty months after the engraftment, established $C 26: 0$ concentration was elevated to about $200 \%$ as compared to the first diagnosis result (Table I). In this case, the detected increasing VLCFA levels were accompanied by a decline in chimaerism.
Very long-chain fatty acids levels following the second haematopoietic stem cell transplantation decreased slightly but were maintained at the level characteristic of hemizygotes.

When the XX chimaerism was lower than $50 \%$, all VLCFA parameters increased even over the diagnostic levels (Fig. 1; Table I). The VLCFA levels showed 
Table I. Chimaerism and $\mathrm{C} 26: 0[\mu \mathrm{g} / \mathrm{ml}]$ and C24:0/C22:0 ratio after the second haematopoietic stem cell transplantation in an X-linked adrenoleukodystrophy patient

\begin{tabular}{|ccccc|}
\hline $\begin{array}{c}\text { After the } \\
\text { second } \\
\begin{array}{l}\text { HSCT } \\
\text { (months) }\end{array}\end{array}$ & \multicolumn{2}{c}{$\begin{array}{c}\text { Chimaerism [\%] } \\
\text { XX }\end{array}$} & $\begin{array}{c}\text { C24:0/ } \\
\text { C22:0 }\end{array}$ & $\begin{array}{c}\text { C26:0 } \\
{[\mu \mathrm{g} / \mathrm{ml}]}\end{array}$ \\
\hline 1 & 100 & 0 & 1.558 & 0.977 \\
\hline 2 & 95 & 5 & 1.376 & 0.817 \\
\hline 2.5 & 97 & 3 & 1.579 & 0.819 \\
\hline 3.5 & 85 & 15 & 1.509 & 0.711 \\
\hline 5 & 55 & 45 & 1.457 & 0.509 \\
\hline 8 & 32 & 68 & 1.741 & 0.916 \\
\hline 12 & 34 & 66 & 1.752 & 1.600 \\
\hline 15 & 25 & 75 & 1.848 & 1.200 \\
\hline
\end{tabular}

a negative correlation with chimaerism, the highest for C24:0/C22:0 ( $r=0.991)$.

One and a half years after the second HSCT, because of the increase in serum VLCFA levels, Lorenzo's Oil was restarted in the patient, which in turn resulted in a repeated decline in very long-chain fatty acids levels (Fig. 1).

At the age of 7 years, the patient was clinically and radiologically asymptomatic, although signs of adrenal insufficiency were noted (test with Synacthen; ACTH - $441 \mathrm{pg} / \mathrm{ml}$ ). The supplementation of adrenocortical insufficiency was started using hydrocortisone $2 \times 5 \mathrm{mg} /$ daily.

At the age of 11 years and 5 months, the patient showed the first signs of cerebral demyelinisation in MRI.

\section{Discussion}

Very long-chain fatty acids levels are the characteristic biochemical biomarkers of X-linked adrenoleukodystrophy but their role as a pathogenic factor is still unclear. Due to the continuing lack of markers that can predict the phenotype of the disease, it is difficult to reliably assess the application of treatment methods in asymptomatic patients. In this study we monitored VLCFA levels in X-linked adrenoleukodystrophy in an asymptomatic patient treated with Lorenzo's Oil or haematopoietic stem cell transplantations.
Our data showed that the most effective decrease in very long-chain fatty acids serum levels was observed during Lorenzo's Oil administration. The VLCFA levels in a patient decreased to the normal range, similar as in patients described earlier [10]. Although it is difficult to show a clinical effect in asymptomatic patients, Moser et al. found an association between a reduction in VLCFA plasma concentration and the development of the childhood cerebral form of X-linked adrenoleukodystrophy [5]. However, previous studies with Lorenzo's Oil have not shown relevant clinical effects $[13,15]$.

Similarly, as has been observed earlier [7], in our study, immediately after the engraftments, a large increase in VLCFA levels was found. This could be a result of the application of the necessary transplantation procedure.

From 6 to 8 months after the transplantation, the VLCFA decreased to the heterozygote levels as demonstrated in Fig. 1. Previous reports revealed that in the case of successful haematopoietic stem cell transplantation, VLCFA serum is decreased but not normalized and maintained at the heterozygote level $[7,9,11,14]$.

The analysis of very long-chain fatty acids parameters showed a correlation between the chimaerism after haematopoietic stem cell transplantation and a serum VLCFA level.

In our study we found, at 6-8 months after the $\mathrm{HSCT}$, that chimaerism correlated negatively with very long-chain fatty acids serum levels and the increasing VLCFA level indicates rejection of the transplant. On the contrary, CD3 in lymphocytes T and very long-chain fatty acids shows no correlation.

Previously it was suggested that analysis of very long-chain fatty acids levels after haematopoietic stem cell transplantation is not indispensable [6]. Although it is true that we still do not know the exact pathological mechanisms in adrenoleukodystrophy, recent studies shed more light on the role of VLCFA. Hein et al. showed the very long-chain fatty acids cytotoxic effect on the nervous system. The increased VLCFA concentrations induce cell death in oligodendrocytes and astrocytes and also deregulation of intracellular calcium homeostasis. Other investigations revealed that VLCFA take part in reactive oxygen species formation $[3,4,12]$. This evidence, just as our results demonstrated above, tends to conclude that VLCFAs should be controlled after any therapeutic approach in X-linked adrenoleukodystrophy patients. 
The presented data showed that the sequential monitoring of very long-chain fatty acids serum levels after haematopoietic stem cell transplantation is important for assessment of engraftment, graft failure or rejection, and could be useful in the treatment effectiveness.

\section{Disclosure}

\section{Authors report no conflict of interest.}

\section{References}

1. Berger J, Gartner J. X-linked adrenoleukodystrophy: clinical, biochemical and pathogenetic aspects. Biochim Biophys Acta 2006; 1763: 1721-1732.

2. Cartier N, Aubourg P. Hematopoietic stem cell transplantation and hematopoietic stem cell gene therapy in X-linked adrenoleukodystrophy. Brain Pathol 2010; 20: 857-862.

3. Fourcade S, Lopez-Erauskin J, Galino J et al. Early oxidative damage underlying neurodegeneration in X-adrenoleukodystrophy. Hum Mol Genet 2008; 17: 1762-1773.

4. Hein S, Schonfeld P, Kahlert S, Reiser G. Toxic effects of X-linked adrenoleukodystrophy associated, very long chain fatty acids on glial cells and neurons from rat hippocampus in culture. Hum Mol Genet 2008; 17: 1750-1761.

5. Moser HW, Raymond GV, Lu SE, Muenz LR, Moser AB, Xu J, Jones RO, Loes DJ, Melhem ER, Dubey P, Bezman L, Brereton NH, Odone A. A Follow-up of 89 asymptomatic patients with adrenoleukodystrophy treated with Lorenzo's oil. Arch Neurol 2005 62: 1073-1080.

6. Peters C, Charnas LR, Tan Y et al. Cerebral X-linked adrenoleukodystrophy: the international hematopoietic cell transplantation experience from 1982 to 1999. Blood 2004; 104: 881-888.

7. Shapiro E, Krivit W, Lockman L, Jambaqué I, Peters C, Cowan M, Harris R, Blanche S, Bordigoni P, Loes D, Ziegler R, Crittenden M, Ris D, Berg B, Cox C, Moser H, Fischer A, Aubourg P et al. Longterm effect of bone-marrow transplantation for childhood-onset cerebral X-linked adrenoleukodystrophy. Lancet 2000; 356 713-718.

8. Shimozawa N. Molecular and clinical findings and diagnostic flowchart of peroxisomal diseases. Brain Dev 2011; 33: 770-776

9. Stradomska TJ, Tylki-Szymańska A. Serum very long-chain fatty acids levels determined by gas chromatography in the diagnosis of peroxisomal disorders. Folia Neuropathol 2009; 47: 306-313.

10. Stradomska TJ, Tylki-Szymańska A. Examination of very long chain fatty acids in diagnosis of $\mathrm{X}$-linked adrenoleukodystrophy. Pediatr Pol 1996; 71: 197-201.

11. Stradomska TJ, Tylki-Szymańska A. Adrenoleukodystrophy (X-ALD) - changes in serum VLCFA profile and clinical course of patients following bone marrow transplantation and administration of Lorenzo oil. Eur J Paediatr Neurol 2008; 12: S73.

12. Stradomska TJ. Peroksysomy - funkcje i zaburzenia metaboliczne. Post Biochemii 2011; 57: 183-190.

13. Suzuki Y, Imamura A, Shimozawa N, Kondo N. The clinical course of childhood and adolescent adrenoleukodystrophy before and after Lorenzo's oil. Brain Dev 2001; 23: 30-33.
14. Suzuki Y, Isogai K, Teramoto T, Tashita H, Shimozawa N, Nishimura M, Asano T, Oda M, Kamei A, Ishiguro H, Kato S, Ohashi T, Kobayashi H, Eto Y, Kondo N. Bone marrow transplantation for the treatment of X-linked adrenoleukodystrophy. $J$ Inherit Metab Dis 2000; 23: 453-458.

15. van Geel BM, Assies J, Haverkort EB, Koelman JH, Verbeeten B Jr, Wanders RJ, Barth PG. Progression of abnormalities in adrenomyeloneuropathy and neurologically asymptomatic in X-Linked adrenoleukodystrophy despite treatment with "Lorenzo's oil”. J Neurol Neurosurg Psychiatry 1999; 67: 290-299. 